\title{
Field test of a lidar wind profiler
}

\author{
Gerard J. Kunz \\ TNO-Physics and Electronics Laboratory \\ P.O.Box 96854, 2509 JG The Hague, The Netherlands
}

\begin{abstract}
Atmospheric eddies, which have slightly different properties than their environment and are believed to be transported by the wind (Taylor's hypothesis), are used as tracers for remote wind measurements with a fast incoherent lidar. Horizontal measurements, parallel with the wind, have shown that the atmospheric structures can be traced in space and time and that the horizontal wind speed can be determined from a set of subsequent measurements. Also, the characteristic size and life time of the structures were inverted from the lidar data. In a second phase, the horizontal wind vector was measured using a synchronously operating dual lidar system with their axes pointing in slightly different horizontal directions. Using the cross-correlation technique, the wind vector was derived from the geometry of the system and the transient times of the structures crossing the two lidar field-of-views. The measured wind vectors are comparable with the in situ measured wind vector. The same technique was applied to measure the vertical profile of the wind vector using a single lidar in the triangulation mode using a fast adjustable platform pointing subsequently in three different (azimuth and elevation) directions. The wind vector could be measured to altitudes of about $1 \mathrm{~km}$ and were in agreement with the in situ measured data from sensors on a $200 \mathrm{~m}$ high meteo mast and from data provided by a Doppler sodar.
\end{abstract}

\section{INTRODUCTION}

In 1985, TNO Physics and Electronics Laboratory developed a $10 \mathrm{~Hz}$, scanning dual lidar ${ }^{1}$ with direct detection, a.o. for atmospheric studies and for cloud mapping purposes. During several experiments, it was noticed that sometimes, even under the clearest conditions, (invisible) structures were detectable in the atmospheric mixed layer. These structures might have been caused by differences in aerosol concentration but also by spatial variations of the air composition or the refractive index ${ }^{2}$. Generally, it is assumed that the atmospheric structures drift with the wind (Taylor's hypothesis). Therefore they can be used as a tracer for wind measurements both in the horizontal plane and vertically for remote sensing of the wind vector as a function of altitude. In an earlier work ${ }^{3}$, some preliminary experimental results were compared with data from an in situ wind sensor mounted on a small meteo tower about $8 \mathrm{~m}$ above the ground. However, no data was available for comparison at higher altitudes. In October/November 1992, we had the opportunity to carry out lidar measurements in the direct vicinity of the $213 \mathrm{~m} \mathrm{high}$ meteo mast ${ }^{4}$ of the Royal Netherlands Meteorological Institute, KNMI, at Cabauw in The Netherlands. At that time, the mast was instrumented at 10, 20, 40, 80, 140 and 200 $\mathrm{m}$ above ground. Especially for this experiment, KNMI operated a Doppler sodar ${ }^{5}$ for measuring the wind vector with a $25 \mathrm{~m}$ vertical resolution to maximum altitude $525 \mathrm{~m}$, depending on the atmospheric reflectivity.

During the Cabauw experiment, the horizontal wind vector could be remotely measured to ranges of about $1.5 \mathrm{~km}$. Measurements at positive elevation angles were carried out in the triangulation mode (three different directions) to determine the wind vector as a function of altitude. The lidar data were compared with data from the wind sensors on the mast and with data provided by the KNMI Doppler sodar. It was shown that the vertical wind profile could be measured to a maximum altitude of about $1 \mathrm{~km}$. The results depend strongly on the availability of detectable atmospheric structures and, obviously, also on the stability of the lidar. 


\section{TEST SITE AND WEATHER}

The Cabauw test site of the Royal Netherlands Meteorological Institute ${ }^{4}$ is located in the Lopik Polder near Cabauw, a small village in the province of Utrecht, between the cities of Utrecht and Rotterdam. The ground is flat, has short-cut grass and occasional trees. Flocks of sheep were grazing on the fields. The site is close to the river Lek and has only some small farm houses in the vicinity. Large cities and heavy industries are not found within a radius of about $20 \mathrm{~km}$. The 213 $\mathrm{m}$ high mast has a diameter of about $2 \mathrm{~m}$. At height intervals of $20 \mathrm{~m}$, there are platforms with three booms, proportionally spaced over 360 degrees. Meteo sensors can be mounted on the tips of the booms. During the lidar experiment, the booms at 40,80, 140 and $200 \mathrm{~m}$ were equipped with, among others, Gill propeller vanes type $8002 \mathrm{DX}$. An additional wind sensor at a different but smaller mast provided the wind data at altitudes of 10 and $20 \mathrm{~m}$. Half-hour means and standard deviations of the meteo data were made available by KNMI. The KNMI Doppler sodar was installed in the meadow about $200 \mathrm{~m}$ north of the mast. This sensor provided the wind vector to maximum altitudes of about $525 \mathrm{~m}$ with a vertical resolution of $25 \mathrm{~m}$ and averaged over 20 minutes.

During the experiments, the wind was mainly from the west to south-west with a varying force between 3 and 7 Beaufort. Temperature varied between about 4 and $12^{\circ} \mathrm{C}$. Rain (drizzle and showers) was experienced on October 29, November 2 and 10. From November 5 to 9 , early morning fog reduced the visibility to about $1 \mathrm{~km}$.

\section{EXPERIMENTAL RESULTS}

During the experiment at Cabauw, about 250 lidar measurements were carried out in sessions with durations varying from several minutes to about 25 minutes. To see differences over the day, measurements were carried out from before sun rise until after sun set. Parts of the data files could not be used for further analysis, due to the limited signal-to-noise ratio and due to the absence of detectable atmospheric structures.

\section{$\underline{3.1 \text { Horizontal wind measurements }}$}

Horizontal wind measurements with an incoherent lidar, parallel to the wind direction, are assumed to be simplest. Therefore we started the measurements and the analysis with these types, keeping in mind that only one component of the wind, the wind speed, can be determined in this case. This can be accomplished either by using the relatively complex and time-consuming cross correlation method, or by estimating the displacements in time of the structures in the lidar data. For measurements of the wind vector in the horizontal plane, a second lidar is necessary that measures in a slightly different azimuth direction. With this second lidar, it is possible to measure the transient times of the atmospheric structures that cross the fields-of-view of the two lidars. Because the lidars also measure the ranges toward the structures and because the geometry of the system is known, the horizontal wind vector can be inverted. This was one of the backgrounds for the design and realisation of the Scanning Miniature Automatic Lidar (SMAL) ${ }^{1}$.

\subsubsection{Single lidar measurements}

From successive measurements in the horizontal plane, parallel to the wind direction, the drifting atmospheric structures could be followed both in space and time. These data provide sufficient information to derive the wind speed and to derive the characteristic size and lifetime of the structures. A brief description of the basic principles used are given below.

A coherent set of lidar waveforms is represented as a two dimensional matrix in which the rows represent the range and the columns represent the time. The values of the matrix elements represent the atmospheric backscatter intensity. Either by inter-connecting the matrix elements with equal values using contour lines or by coding the values in a grey scale or in false color, the atmospheric structures and their displacements can be visualized in one figure. It is assumed that the lateral variations of the wind vector are not too large and that the life-time of the structures is long in comparison with the repetition rate of the lidar. In the first step of the analysis, the cross-correlation function is calculated between a backscatter time-series at a certain range (this is one row of the matrix) and a second backscatter time-series at an other range. The time shift of the peak in the cross correlation function provides the time that the atmospheric structures needed to drift from one range to the other, and the wind speed is determined from the ratio of the range difference and the time shift. The size and the life time of the atmospheric structures can be derived from the two dimensional cross correlation 
function which is obtained by cross correlating one backscatter time-series at a certain range with the backscatter timeseries at all other ranges and times. Contour lines of equal cross correlation values, can be represented by concentric ellipses $^{6}$. An hypothetical example of such a correlogram, with the range shift on the horizontal axis and the time shift on the vertical axis is presented in Figure 3.1.

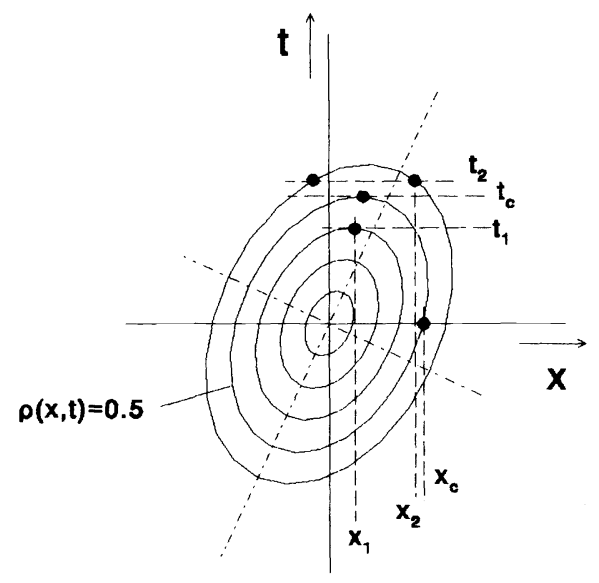

Figure 3.1: Hypothetical results of a two-dimensional cross correlogram (continuous) with the range shift on the horizontal axis and the time shift on the vertical axis

The characteristic size of the structures is given by the intercepts of the contour line with cross correlation value of 0.5 (also called the characteristic ellipse) with the horizontal axes (range shift). This value is indicated with $\mathrm{X}_{\mathrm{c}}$. If there were no drift, the characteristic life time of the structures would be given by the intercept of the same contour line with the vertical axes (time shift). In the presence of drift, however, this location is shifted either to the right or to the left, depending on the direction of the drift. The characteristic time is found from the horizontal tangents to the characteristic ellipse assuming that it does not change under the influence of drift. One value of $\mathrm{t}_{\mathrm{c}}$ has been indicated in Figure 3.1. Apart from the characteristic life time, the ratio of the $\mathrm{x}$ - and $\mathrm{y}$-values of the co-ordinates of $\mathrm{t}_{\mathrm{c}}$ provide the wind speed. It is noted, that the inversion of the wind speed is not limited to the characteristic ellipse alone but can, in principle, be calculated from any contour line that represents a certain cross correlation level (ellipse).

To demonstrate the practical implementation of the cross correlation method, the results of two lidar data files have been presented in contour lines of equal correlation in Figure 3.2. Contour lines have been drawn between points of equal cross correlation in the interval from 0.1 to 0.9 , in steps of 0.2 . The data of November 5, 11:29 a.m., (left) was recorded during hazy conditions. In the right part of the figure, the contour lines of equal correlation are presented of the data measured on November 9, 06:32 p.m., during periods of occasional light drizzle. As a result, the signal-to-noise ratio of the structures is much better. Also the variability of the slopes in the structures is much smaller. During the development and the application of the inversion procedure, it was found that best results were obtained by splitting the data matrix both horizontally and vertically into three sub-figures and calculating a mean cross-correlation matrix from these subdata-sets.

For November 5, 11:29 a.m., the contour lines of equal cross correlation are very poor and the characteristic ellipse with cross correlation value of 0.5 cannot clearly be discriminated due to the noise in the data. Moreover, the instability of the laser resulted in a relatively large correlation for all possible range shifts (small vertical contour) and a destruction of the shape of the ellipses. Therefore, in this example it is not possible to derive the characteristic properties of the structures. The wind speed, on the other hand, can be derived from the contour with cross correlation value of 0.1 . The contour lines of equal cross correlation derived from the data measured on November 9, 06:32 p.m., are presented using the same grid (scaling) parameters as used in the left figure. In this example, the characteristic contour with value 0.5 can clearly be distinguished and the contour lines are relatively parallel. Both the characteristic parameters and the wind speed were derived from the contour lines. 

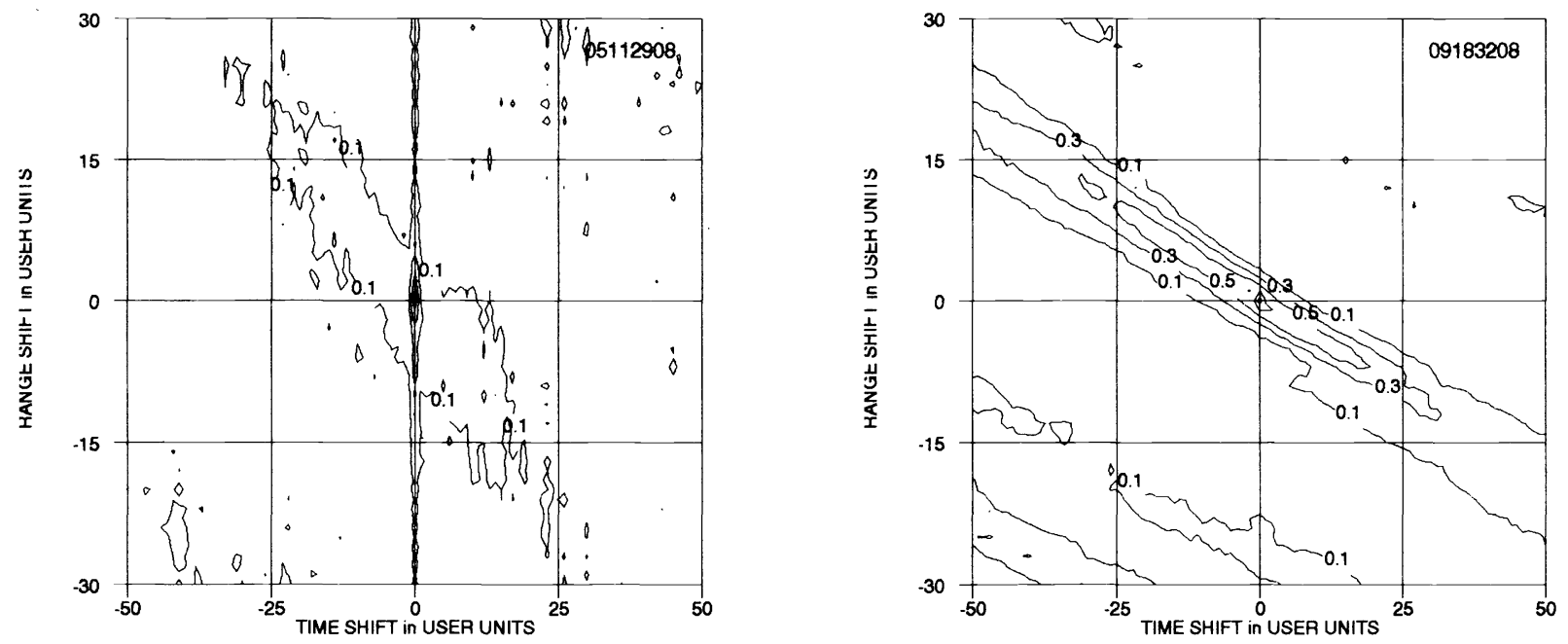

Figure 3.2: Contour lines of equal cross correlation value derived from the data measured on November 5 , 11:29 a.m (left) and on November 9, 06:32 p.m (right).

Following the procedure described above, all the measurements in the horizontal direction, parallel to the wind, were processed. The results of these calculations have been compared with the in situ measured wind speed at an altitude of 20 $\mathrm{m}$ and presented in Figure 3.3.

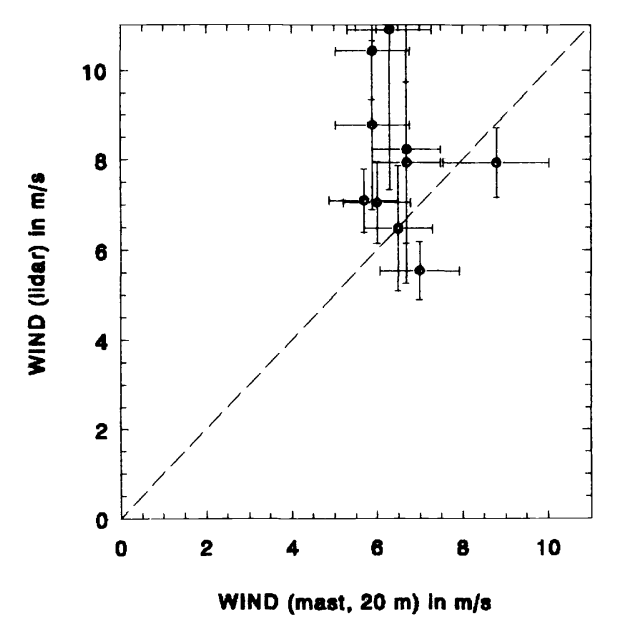

Figure 3.3: Horizontal wind speed results derived from lidar measurements parallel to the wind, using the cross correlation method. The in situ measured wind speed at an altitude of $20 \mathrm{~m}$ is also shown.

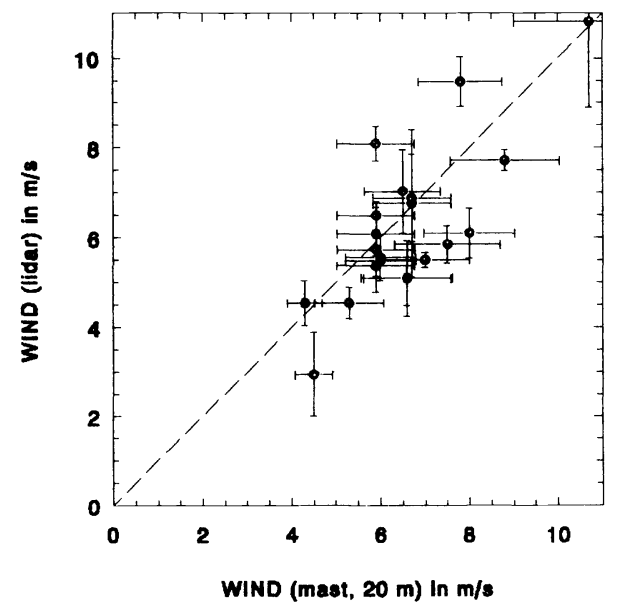

Figure 3.4: Horizontal wind speed results derived from lidar measurements parallel to the wind, using the slopes of the structures in the false color representation of the atmospheric backscatter structures. The in situ measured wind speed at an altitude of $20 \mathrm{~m}$ is also shown 
Inversion of the wind speed from lidar measurements parallel to the wind based on cross-correlation, during the Cabauw experiment has provided reasonable results in the wind speed interval between about 6 and $8 \mathrm{~m} / \mathrm{s}$. The wind speeds calculated from the lidar data are higher than provided by the mast. This might be due to the accuracy of the absolute value of the small elevation angle of the lidar and/or due to the integration length of the time series. Because of the very limited wind speed interval, the results are not conclusive. We think that the limited signal-to-noise ratio of the atmospheric reflections and the instability of the laser are reasons for these limited results. The analyses show that the characteristic size and the characteristic life time of the structures, in this limited wind speed interval, varied from zero to about $50 \mathrm{~m}$ and $2 \mathrm{~s}$ respectively. The number of data point is too limited for detailed statistical analysis.

Inspection of false color representations of the lidar data suggested that it would be possible to estimate the slope of the structures in many more cases than could be processed with the cross correlation method. From the displacement in a certain time interval, the wind speed can also be calculated. For proper calculation, the sampling interval of the lidar waveforms and the repetition rate of the lidar must be taken into account. Therefore, a program was developed that could interactively draw lines with adjustable slopes over parts of the false color figures. The average slope and the standard deviation were estimated from 5 to 10 lines fitted over parts of the figure that had sufficient structure. These results are again compared with the in situ measured wind speed at $20 \mathrm{~m}$ altitude and are presented in Figure 3.4.

The results of Figure 3.4 show that the wind speeds derived from the lidar measurements compare well with the in situ measured wind speed at $20 \mathrm{~m}$ altitude. It is therefore concluded that the slopes of the patterns in the false color presentation of horizontal lidar signals, measured parallel to the wind, compare well with the wind speed measured with an in situ cup anemometer at an altitude of $20 \mathrm{~m}$ (correlation better than $83 \%$ ). In addition, this method is more successful than the more complex cross correlation calculations, probably because the structures are manually selected with more intelligence than the automatic cross correlation procedure. Possibly, the cross correlation can be improved by interactive selecting areas with much structure.

\subsubsection{Dual lidar measurements}

In the previous section it was shown that the horizontal wind speed could be inverted from single-lidar measurements parallel to the wind direction. (Thus one property of the wind vector was determined with a single lidar using a priori information.) Using two simultaneously operating lidars, or a single lidar that can probe the atmosphere alternately (with sufficient high frequency) in two different horizontal directions, both the horizontal wind speed and the wind direction can be determined as a function of range. The principle is based on determining the transient time of the atmospheric structures that cross the fields of view of the two lidars (cross wind). The distance travelled can be derived from the geometry of the system and the range information available in the lidar signals. The ratio of the distance and the transient time provides the wind speed.

This procedure requires cross-correlations of a huge amount of data, which is time consuming. Therefore, an adaptive program was developed that controls the size of the field based on the maximum value of the cross correlation, calculated in a previous step. The evolution of the algorithm is followed by presenting the results in false color figures. The feedback criteria depended on the amount of structure in the data and the signal-to-noise ratio.

As an example for this procedure, the data measured on November 2, 12:09 p.m. (file 02120923) and some of the intermediate results, are presented. In Figure 3.5, the transient times and distances from the second lidar are presented as a function of the range bin number of lidar one. Both parameters increase linearly with the range bin number of lidar one because the lidars operate from the same location but under a different azimuth angle. To each set of data points, a straight line through the origin can be fitted. This indicates that the inversion procedure works properly. 


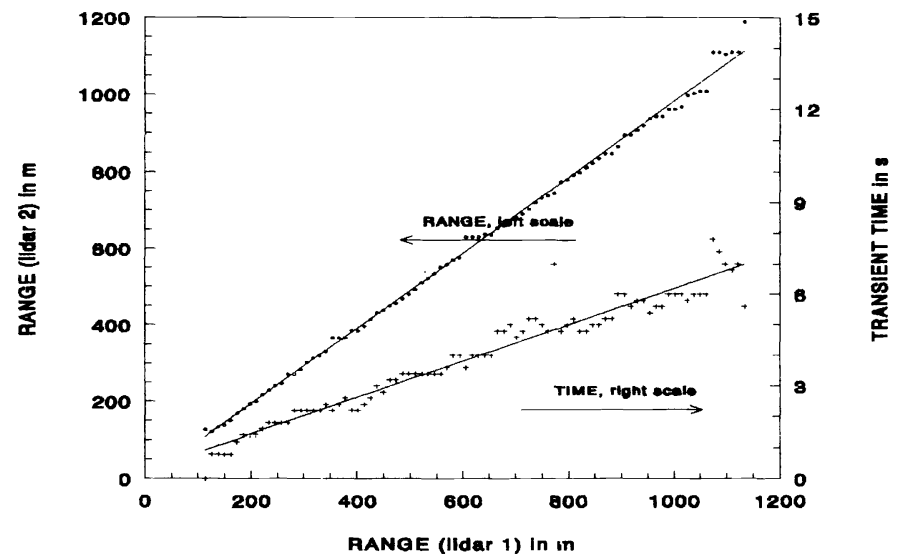

Figure 3.5: The location were the atmospheric structures crossed the axis of the second lidar (solid dots) as a function of the location were these structures crossed the axis of the first lidar. The crosses indicate the transient times.

During the Cabauw experiment, we recorded 11 successful dual lidar horizontal measurements with sufficient structure for remote sensing of the (horizontal) wind vector. 10 of these files provided sufficient signal-to-noise ratio for further analysis. The results of the analyses are presented in Figure 3.6.
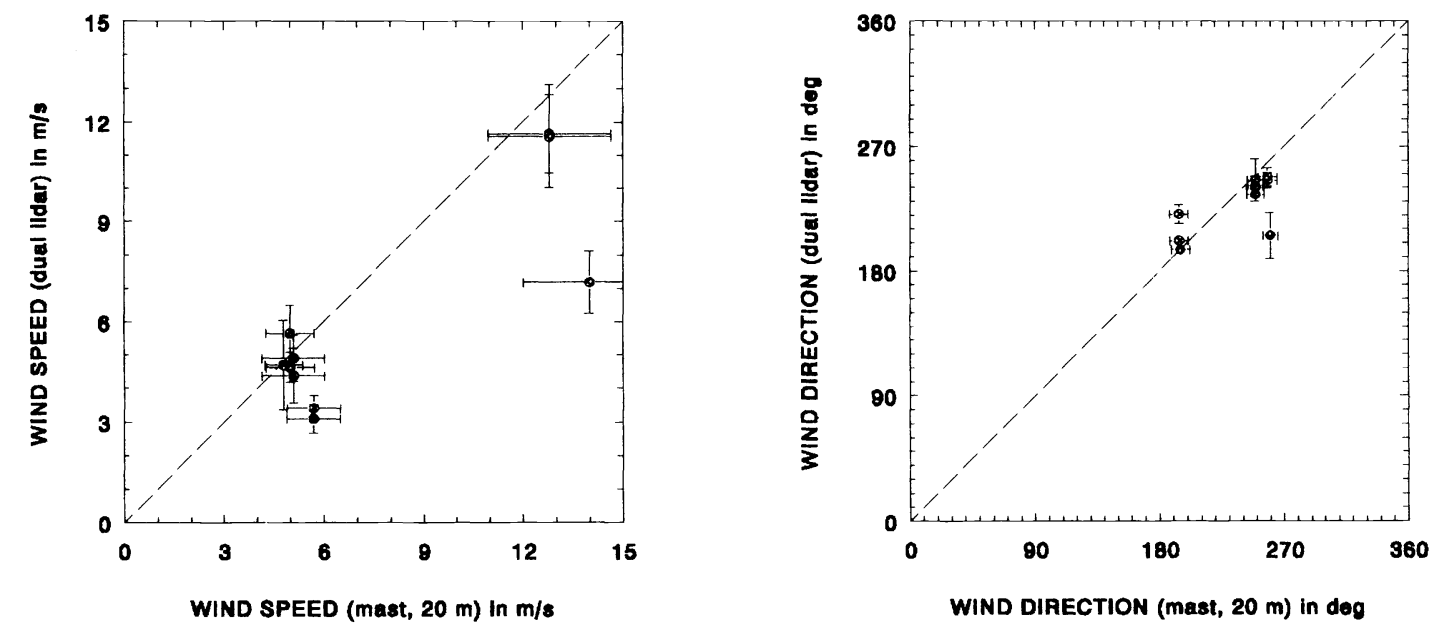

Figure 3.6: Wind speed and wind direction derived from dual lidar measurements, in the horizontal plane, as a function of the in situ measured data at $20 \mathrm{~m}$ altitude.

The data points in the Figure 3.6 are clustered in two groups (because of the atmospheric condition and not due to the direction of the lidar). There is a good correlation, about $81 \%$, for both the wind speed and the wind direction, between the remotely sensed wind vector and the in situ measured wind vector at $20 \mathrm{~m}$.

It is concluded that the horizontal wind vector can be obtained from measurements with the dual lidar system. The relatively long inversion time (about 10 minutes per range profile on a $386 \mathrm{PC}$ ) can be reduced by optimizing the inversion scheme andior by using a faster computer. 


\subsection{Slant path measurements}

Turbulent structures are detectable with an incoherent lidar due to the spatial variation in aerosol concentration and/or differences in the air composition. Variations in refractive index are mainly caused by differences in either the temperature and/or the water vapor content. Assuming that these structures drift with the wind (Taylor's hypothesis), they can in turn serve as tracers for wind profile measurements using an incoherent lidar. The principle is based on mapping the drifting atmospheric structures using three simultaneously operating lidars in different elevated directions. The principle is not new. It has been used for many years by different institutes ${ }^{7,8}$. Since a triple lidar system was not available for our experiments, we simulated it with one lidar. The single TNO SMAL lidar was mounted on a rapidly adjustable platform for consecutive operation in the three desired directions. A set of triangulation lidar measurements takes about 25 minutes. Part of this time was required for the measurements itself and another, not insignificant, part of the time was needed to adjust and stabilize the platform in the desired directions. Previous measurements ${ }^{3}$ indicated that only in a limited number of cases sufficient atmospheric structure was present to perform wind measurements with lidar. Therefore, prior to the triangulation measurements, measurements were made at a fixed elevated direction to determine whether such a measurement would be useful. This resulted in a relatively large fraction of useful data. Despite of a damaged Pockelscell (optical switch in the laser) we recorded 25 triangulation measurements from which 13 could be used for further analysis.

\subsubsection{Description of the inversion process}

The initially applied inversion process was based on finding the maximum cross correlation value of a backscatter time series at a certain height measured by lidar-1 with a larger number of backscatter time series over a certain height interval measured by the other two lidars. As a result, the inversion procedure had the freedom to find the best fit within reasonable height limits to invert the three dimensional wind vector. However, application of this procedure provided occasionally unrealistic vertical wind speeds. Therefore, it was decided to cross correlate only the backscatter time series provided by the three lidars in a small height interval assuming that the mean vertical wind component, averaged over about 15 minutes, is zero. This resulted in better correspondence with the mast data and the data from the Doppler sodar and in addition in a shorter inversion procedure.

\subsubsection{Wind vector as a function of altitude}

Typical wind profiles, obtained from the lidar measurements at Cabauw, will be presented and discussed in this section. These examples have been selected because they showed a particular profile for wind speed and/or for wind direction.

Wind profile on October 30, 03:25 p.m.

This triangulation lidar measurement started at 03:25 p.m. and lasted until 03:51 p.m. During the measurements, a cloud deck moved in, but no precipitation was observed. The lidar data exhibited only a few large structures to an altitude of about $700 \mathrm{~m}$, in all three measuring directions. Data from the in situ wind sensors on the mast were available from the levels 10, 20, 40,80,120 and $200 \mathrm{~m}$. The Doppler sodar provided the wind vector up to $300 \mathrm{~m}$ in steps of $25 \mathrm{~m}$ and an altitude of $375 \mathrm{~m}$. The results obtained with the lidar and those obtained from the other sensors are presented in Figure 3.7. The vertical solid line is a fictive representation of the mast and is drawn for reference only. The solid dots represent the lidar results and the triangles represent the results from the Doppler sodar. The plus signs represent the residual vertical wind component. Part of the lidar data at low altitudes is missing due to the geometric set-up of the lidar (laser and the receiver bi-static). Also, between 125 and $200 \mathrm{~m}$ altitude, there is a relatively large difference between the lidar derived results and the data from the mast. This is caused by the geometry of the triple lidar and by subsequently measuring in the three directions. As a result, the distances between the axes of lidar- 1 and lidar-2, and between lidar- 1 and lidar-3, at these heights, are relatively small compared with the product of transient time and drift speed. Consequently, the time series of the atmospheric structures at these low altitudes are under-sampled providing erroneous input data for the inversion procedure. Above $200 \mathrm{~m}$, however, the lidar data follow the trend of the wind direction data from the sensors on the mast and the data from the Doppler sodar. 

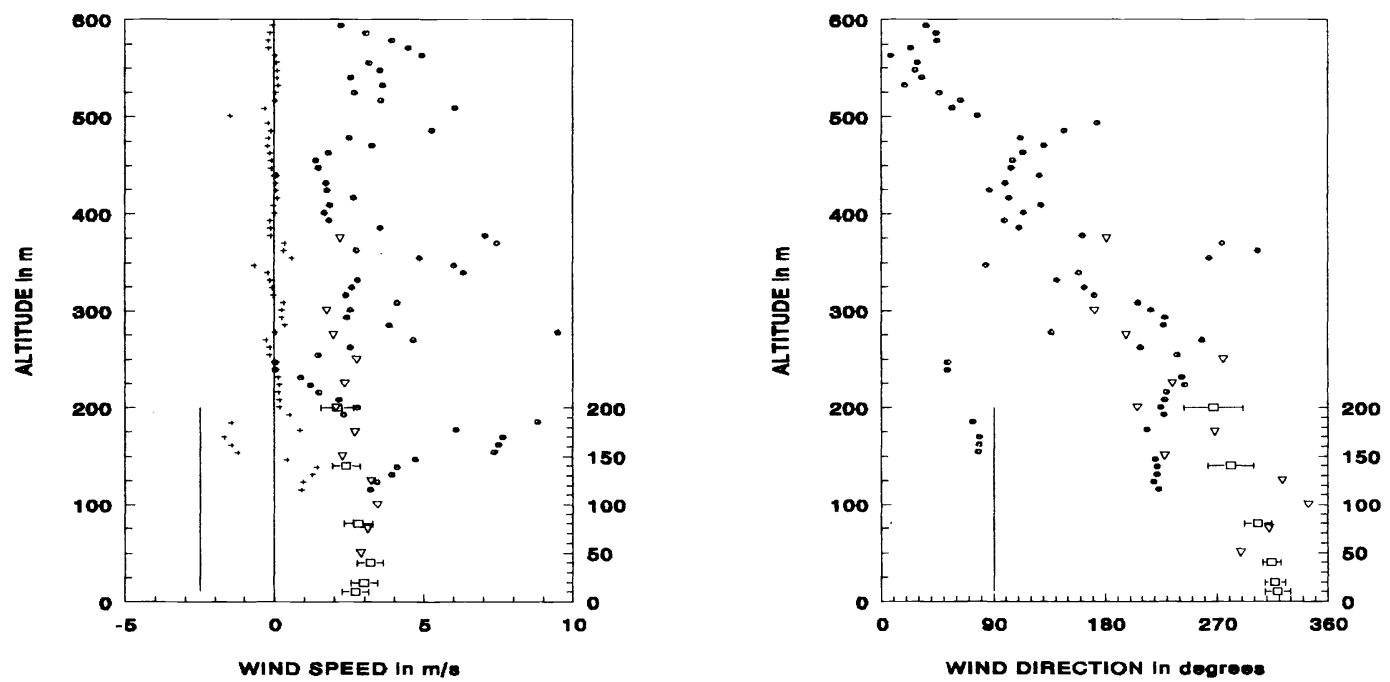

Figure 3.7: Profiles of wind speed (left figure) and wind direction (right figure) derived from triangulation lidar measurements (solid dots). Also shown are the profiles from sensors on the mast (open squares with error bar) and from Doppler sodar (open triangles). The thin plus signs represent the vertical wind speed determined from the lidar. Measurements on October 30, 1992, 03:25 p.m.

Wind profile on November 3, 10:36 a.m.

This wind profile measurement started on 10:36 a.m. and lasted until 11:01 a.m. During this time interval, the sky was overcast with stratus clouds. There was no precipitation. The lidar detected a clear mixed layer (with increasing scattering as a function of altitude) but with a limited amount of structure. Wind profiles derived from the lidar data and those provided by the other sensors are presented in Figure 3.8. As discussed above, the lidar did not provide reliable results at altitudes below about $200 \mathrm{~m}$. Above that height, the wind profile (speed and direction) compares well with the data from the sensors on the mast and with the data from the Doppler sodar. Note the perfect fit of the wind direction provided by the different sensors. The anomalies in the lidar data between 480 and $550 \mathrm{~m}$ are probably caused by the poor signal-tonoise ratio in the data.
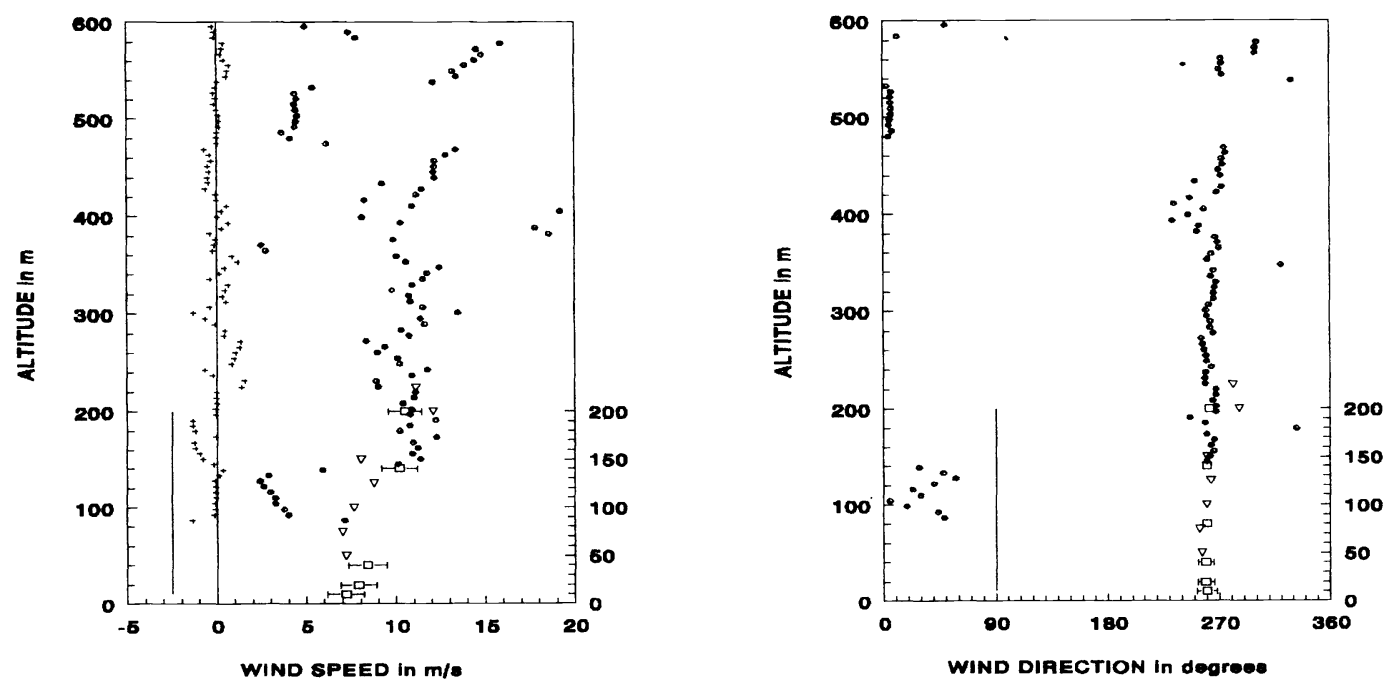

Figure 3.8: Profiles of wind speed (left figure) and wind direction (right figure) derived from triangulation lidar measurements (solid dots) on November 3, 1992, 10:36 a.m. Also are shown the profiles from sensors on the mast (open squares with error bar) and from Doppler sodar (open triangles). The thin plus signs represent the vertical wind speed provided by the lidar data inversion procedure. 
Wind profile on November 3, 11:33 a.m.

On November 3, 1992, from 11:33 a.m. to 11:59 a.m., the lidar measured a wind profile under an overcast sky with occasionally light rain. Due to the large backscatter properties of the rain drops, the structures could be detected with a relatively large signal-to-noise ratio. The wind profile derived from the lidar data, the profiles provided by the sensors on the mast and by the Doppler sodar are shown in Figure 3.9.

The results in Figure 3.9 show that the wind speed profile measured with the lidar follows the profiles from the other sensors. The wind direction on the other hand, shows a systematic difference. At low altitudes, there is again the influence of under sampling of the time series of the atmospheric structures and at the largest altitudes there is the problem of the low signal-to-noise ratio.

It is concluded that the vertical wind profiles, derived from triangulation measurements, compare in general well with the profiles provided by the sensors on the $213 \mathrm{~m}$ high mast and by the KNMI Doppler sodar. TNO's SMAL lidar measured wind profiles from about $200 \mathrm{~m}$ to about $900 \mathrm{~m}$ altitude. The best results were obtained when the time series from identical heights, or from a small height interval at the same level, were taken for analysis. The initially proposed method to also derive the vertical wind component did not provide credible results in many occasions (on average the vertical wind component must be zero).
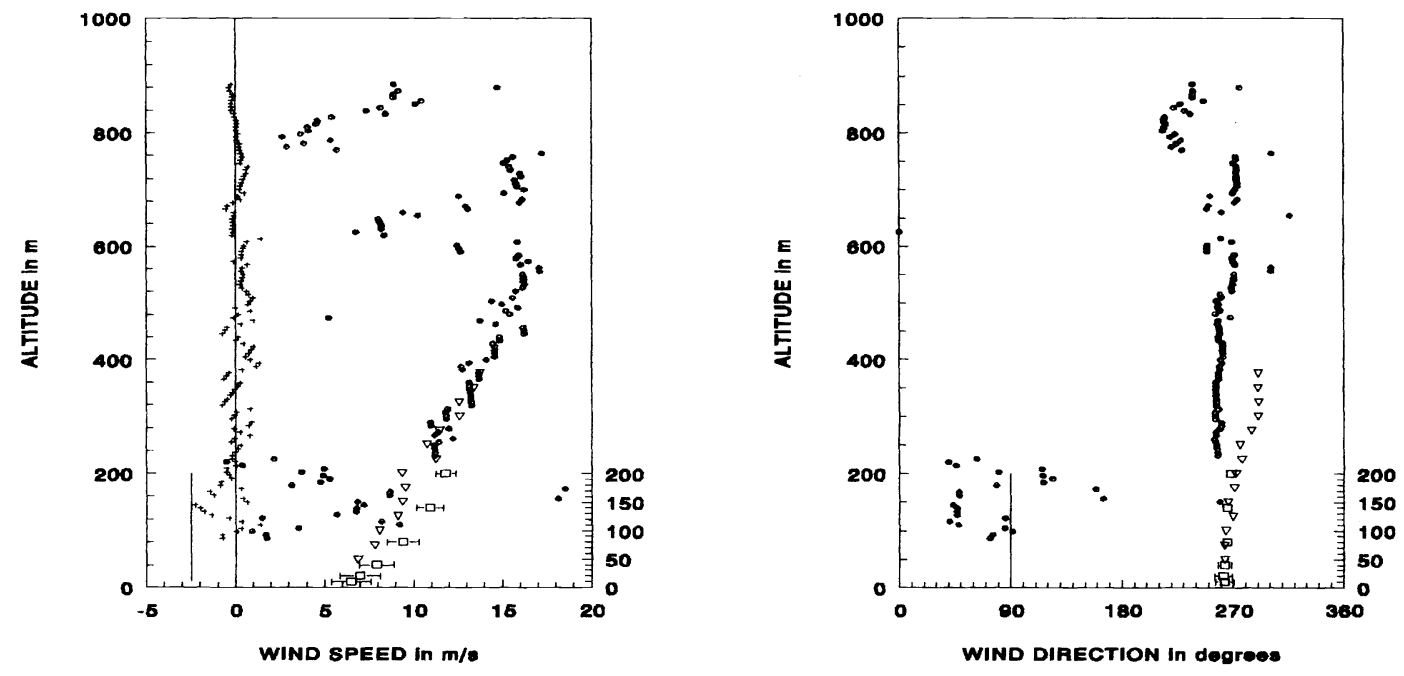

Figure 3.9: Profiles of wind speed (left figure) and wind direction (right figure) derived from triangulation lidar measurements (solid dots). Also are shown the profiles from sensors on the mast (open squares with error bar) and from Doppler sodar (open triangles). The thin plus signs represent the vertical wind speed provided by the lidar data inversion procedure. Measurements on November 3, 1992, 11:33 a.m.

Because we did not have a possibility to measure simultaneously in three different directions, the measurements were consecutively performed in the three desired directions. As a result, data are missing in at least $67 \%$ of time. In practice, this is even worse because the adjustment of the platform takes also some time. These limitations are of minor importance at higher altitudes where the transient times are relatively long due to the relatively large distances between the sensed volumes. At low altitudes, the results are not satisfactory which is due to under sampling of the time series. The problems at low altitudes could be solved somewhat by measuring under larger azimuth angle differences and/or under smaller elevation angles (in exchange of giving up performance at larger heights). 


\section{CONCLUSIONS}

Remote sensing of the wind speed and wind direction with an incoherent lidar depends on the presence of detectable atmospheric structures which are assumed to drift with the wind. These structures occur during the dynamic process of turbulent and/or mechanical mixing of the air in the lowest few kilometers of the atmosphere. Eddies with different aerosol concentration and/or different refractive index might also be responsible for spatial/temporal variation in the atmospheric backscatter. A lidar that can resolve the smallest variations in the atmospheric backscatter with sufficient signal-to-noise ratio requires a very stable laser with a repetition rate of about $10 \mathrm{~Hz}$ or higher, a low-noise receiver with a large dynamic range and a recording system with sufficient resolution and capacity.

To test the hypothesis that the atmospheric structures drift with the wind and can therefore be used as tracer for the wind measurements with an incoherent lidar, we started with lidar measurements parallel to the wind using a priori information on the wind direction. This type of measurement was assumed to be the least complex task with which we could gain experience required to attack more complicated problems such as the two and the three dimensional wind vector. In a first attempt, the horizontal wind speed was calculated by cross correlating consecutive time series from selected ranges. However, it appeared that this automatic method was only effective in a limited number of cases. Therefore, the wind speed was also derived from the slopes of the structures which were visualized by coding the atmospheric backscatter in false color, in range versus time figures. This method appeared to be more successful than the cross correlation calculations. A correlation coefficient of $81 \%$ was found between the lidar derived wind speed and the in situ measured wind speed at an altitude of $20 \mathrm{~m}$.

Apart from the wind speed, sets of consecutive horizontal lidar data, measured parallel to the wind, provide also the characteristic size and the characteristic life time of the structures. This information might be important for estimating the influence of turbulence on imaging systems, provided that the lidar-measured structures are similar to those distorting images. In theory, the characteristic size is defined as the full width half maximum value of the auto correlation function of the momentary (back)scattering along a certain path. Thus in principle from one lidar measurement the characteristic life time is defined as the full width half maximum value of the auto correlation function of the atmospheric (back)scattering time series at a fixed location, in the absence of drift. Because the structures drift with the wind, in general, the characteristic size can only be derived from a set of consecutive lidar measurements. During the Cabauw experiment, characteristic sizes of about $50 \mathrm{~m}$ have been found and characteristic life times of about 2 seconds.

The next logical step was to determine the horizontal wind direction without $a$ priori information. This was done by parallel operation of a real second lidar system under a different azimuth angle. A dual lidar was constructed by mounting a second receiver on top of the main lidar and aligning part of the tapered laser beam parallel to the axis of the second receiver. One extra degree of freedom of the horizontal wind vector (wind direction) is obtained by adding one extra lidar. The wind direction at a certain range was determined from the geometry of the system and the ranges at which identified atmospheric structures crossed the axes of the lidars. The transient time was determined from the time labels coupled to the lidar signals. The ratio of the distance and the transient time provided the wind speed.

In a final step, attempts were made to measure the vertical wind profile assuming that drifting atmospheric structures can also be measured throughout the mixed layer and also in the entrainment layer (altitude between about 1 and $2 \mathrm{~km}$ ). This can be realized with three simultaneously operating lidars each pointing in a different elevated direction. The time series of the atmospheric structures, measured at the corner points of a fictive horizontal triangle at a selected height, are used to determine the transient times of the atmospheric structures crossing the axes e.g. from lidar 1 to lidar 2 and from lidar 1 to lidar 3. From these times, the altitude and the geometry of the triple lidar, the wind vector at that height can be calculated. The vertical wind profile was obtained by repeating this procedure for all the range (altitude) bins in the lidar signals.

The technical complexity of a triple lidar and the inherent costs have led us to simulate such a system by operating a single lidar from a rapidly adjustable platform. This provided us the flexibility to select the desired elevation and azimuth angles. The disadvantages of such a scanning system are that the atmospheric structures are not simultaneously measured and that during the adjustment of the platform, no data can be obtained. This resulted in under-sampling of the time series of the atmospheric structures at iow altitudes because the iidar cannot follow the eddies at short ranges. As a result, the 
wind profile data is not reliable in about the lowest $200 \mathrm{~m}$. In practice, this problem can partly be solved by increasing the azimuth angles between the axes and/or by measuring at lower elevation angles (increasing the range for the same altitude). However, this would reduce the quality and/or the measurements at larger altitudes.

Initially, the procedure to invert the 3-D wind profile from a set of triangulation lidar measurements had the freedom to cross correlate one time series at a certain height from the first lidar with all the time series of the second and the third lidar from all heights. In this way, also the vertical component of the wind vector could be derived. However, it appeared that unrealistic large vertical wind speeds were obtained with very small horizontal wind components. This problem was solved by limiting the vertical extend of the altitude window in the data from lidar- 2 and lidar- 3 .

In order to check the wind profiles obtained by the lidar, we operated the TNO-FEL SMAL lidar in the vicinity of the $213 \mathrm{~m}$ high meteo mast of the Royal Netherlands Meteorological Institute (KNMI) at Cabauw, the Netherlands. During the experiment, the mast was instrumented among others with Gill propeller vanes at 10, 20, 40, 80, 140 and $200 \mathrm{~m}$. Especially for this intercomparison, KNMI also operated the Doppler sodar which provided profiles of the wind to maximum altitudes of about $525 \mathrm{~m}$.

25 triangulation measurements were made. Due to either the limited amount of detectable atmospheric structures and/or due to laser instabilities, useful results were obtained in about $50 \%$ of the cases. It has been shown that the lidar derived vertical wind profiles compare well with the wind profiles measured by the sensors on the mast and by the Doppler sodar. The lidar was operated under a horizontal elevation angle of about 50 degrees. Wind speed and direction were measured to altitudes of about $900 \mathrm{~m}$. By pointing the lidar at larger elevation angles, this altitude can be increased somewhat.

We conclude that it is possible to measure the vertical profile of the horizontal wind vector in the atmospheric boundary layer to altitudes of about $1 \mathrm{~km}$ using TNO's SMAL lidar. However, above the top of the boundary layer and/or above cloud decks, this kind of measurement cannot be made because no structures have been detected (so far). The performance of a lidar wind profiler depends critically on the presence of the atmospheric structures. Conditions under which these structures occur have not been identified.

\section{ACKNOWLEDGEMENTS}

The author realizes that the experiment in Cabauw was an unique opportunity to compare the lidar measured wind profiles with the data from in situ sensors on the $213 \mathrm{~m}$ high mast and with the results from the Doppler sodar. Therefore, in the first place, the author thanks the people of the Royal Netherlands Meteorological Institute (KNMI), especially Dr. A.P. van Ulden for providing the opportunity for this field test, and W.A.A. Monna and J.G. van der Vliet for the local organisation and for operating the KNMI Doppler sodar and for providing the data from the other KNMI sensors. In addition, Dr. Monna is acknowledged for reviewing the manuscript. Furthermore, the author wishes to thank Drs. W. Pelt of the Ministry of Defence for arranging the extra financial support for these measurements. And last but not least I acknowledge Marcel Moerman for his excellent co-operation during these measurements.

The work described in this paper was carried out under contract A90K696 of the Dutch Ministry of Defence and under contract A92KM709 of the Dutch Royal Navy.

\section{REFERENCES}

1. Kunz, G.J., 'A high repetirion rate lidar', TNO-FEL Report, FEL-90-A352, 1991.

2. Owens, J.C., 'Optical refractive index of air: dependence on pressure, temperature and composition', Appl. Opt., 6 (1), , 51-59, 1967.

3. Kunz, G.J., 'Wind measurements with an incoherent lidar' TNO-FEL Report, FEL-93-A040, 1993.

4. Monna, W.A.A. and J.G. van der Vliet, 'Facilities for research and weather observations on the $213 \mathrm{~m}$ tower at Cabauw and remote locations', KNMI Scientific Report, WR 87-5, ISSN 0169-1651, 1987.

5. Beljaars, A.C.M., 'Verification of Doppler sodar measurements', KNMI Scientific Report 85-2, 1985. 
6. Zuev, V.E., Y.M. Vorevodin, G.G. Matvienko, and I.V. Samokhvalov, 'Investigation of structure and dynamic of aerosol inhomogeneities in the ground layer of the atmosphere', Appl. Opt., 16, (8), 2231-2235, 1977.

7. Briggs, B.H., G.J. Phillips and D.H. Shinn, 'The analysis of observations on spaced receivers of the fading of radio signals', Proc. Phys. Soc. B 63, 106-121, 1950.

8. Hooper, W.P. and E.W. Eloranta, 'Lidar measurements of wind in the planetary boundary layer; the method, accuracy and results from joint measurements with radiosonde and kytoon' J. Climate and Appl. Meteorol., 25, 990-1001, 1986. 